\title{
Spatio-temporal coherence in vertically emitting GaAs-based electrically driven polariton lasers
}

\author{
H. Suchomel, ${ }^{1}$ M. Klaas, ${ }^{1}$ S.Betzold, ${ }^{1}$ P. Gagel, ${ }^{1}$ J. Beierlein, ${ }^{1}$ S. Klembt, ${ }^{1}$ C. Schneider, ${ }^{1,}$ a) and S. Höfling ${ }^{1,2}$ \\ 1) Technische Physik, Wilhelm-Conrad-Röntgen-Research Center for Complex Material Systems, \\ and Würzburg-Dresden Cluster of Excellence ct.qmat, Universität Würzburg, Am Hubland, D-97074 Würzburg, \\ Germany \\ ${ }^{2)}$ SUPA, School of Physics and Astronomy, University of St. Andrews, St. Andrews KY 16 9SS, \\ United Kingdom
}

We report on the implementation of a GaAs-based, vertically emitting electrically pumped polariton laser, operated at cryogenic temperatures. The structure consists of a high-quality factor AlGaAs/AlAs microcavity $(\mathrm{Q}=15,000)$ with two stacks of four GaAs quantum wells and features a Rabi-splitting of $11 \mathrm{meV}$. Polariton lasing manifests by a clear threshold in the input-output characteristics of our device with a sharp drop in the emission linewidth and a continuous blueshift of $0.7 \mathrm{meV}$ above threshold with increasing injection current. We measure spatial as well as temporal coherence of our device in the condensed phase by utilizing interference spectroscopy. Our results clearly demonstrate that electrically driven polariton lasers have promise as monolithic polaritonic source of coherent light.

Exciton-polaritons are bosonic quasi-particles which emerge in the strong coupling regime between an exciton and a photon, which is trapped in a microcavity ${ }^{1}$. Obeying bosonic statistics and reaching a critical density, polaritons can dynamically condense into a macroscopically occupied groundstate ${ }^{2}$. The bosonic condensation of exciton-polaritons yields the emission of coherent light, which is leaked from the microcavity and justifies the term polariton laser. Polariton lasing can occur at particle densities less than traditional inversion based devices $^{3,4}$. Due to the large nonlinearity of the system, which enables e.g. ultra-efficient switching ${ }^{5}$, a number of prototype applications based on optical excitation have been investigated $^{6,7}$. Large bandgap materials like GaN and on top of that recent investigations into new material systems with very stable excitons like organics and $2 \mathrm{D}$-materials allow the operation of polaritonic devices at room temperature ${ }^{8-13}$. For scaling and integration however, advances in electrical injection are needed.

One milestone for electrical injection was achieved with a polariton diode at room temperature ${ }^{14}$. First electrically injected polariton lasing in a vertical emitting design has been studied in the presence of external magnetic fields to enhance light-matter coupling ${ }^{15,16}$. Later, electrically injected polariton lasing up to room temperature has been claimed in GaN-based edge-emitting devices, where the electrical current is not injected through the mirror structures, but instead the contacts are directly connected to the sides of the active zone ${ }^{17,18}$. The edgeemitting design has a number of drawbacks considering the established methods of photonic potential engineering for polaritons ${ }^{19}$, which have recently been utilized to extent the field of polaritonics towards the exploitation of topological photonic and polaritonic state ${ }^{20,21}$, as well as strongly correlated systems ${ }^{22-27}$.

In this letter, we discuss the implementation of an elec-

\footnotetext{
a) Electronic mail: christian.schneider@physik.uni-wuerzburg.de
}

trically driven polariton laser diode, which shows a pronounced non-linearity and build up of spatio-temporal coherence without a magnetic field at low temperature in close analogy to the well-established optically injected GaAs-based polariton laser platform. We provide direct evidence for coherence in our system, both in space and time, which establishes our device at the forefront of engineering advanced polaritonic light-sources and devices.

The microcavity was grown via molecular beam epitaxy on a Si-doped GaAs substrate. The mirrors consist of 36 (30) $\mathrm{Al}_{0.2} \mathrm{Ga}_{0.8} \mathrm{As} / \mathrm{AlAs}$ mirror pairs in the bottom (top) distributed Bragg reflector (DBR), whereas the cavity spacer is a $\lambda / 2$ layer comprising two stacks of four $7 \mathrm{~nm}$ thick GaAs-QWs, embedded in $4 \mathrm{~nm}$ thick $\mathrm{Al}_{0.4} \mathrm{Ga}_{0.6} \mathrm{As}$ barriers. The quantum wells are placed in the antinodes of the electromagnetic field. The bottom mirror is Si-doped (n-type), while the top mirror is C-doped (p-type). The doping concentration was reduced symmetrically towards the cavity spacer from $3 \times 10^{18} \mathrm{~cm}^{-3}$ to $0.5 \times 10^{18} \mathrm{~cm}^{-3}$ to facilitate electrical injection with reduced absorption in the mirrors. To further reduce the differential resistance of the DBR mirrors, $\delta$-doped layers with a density of $1 \times 10^{12} \mathrm{~cm}^{-2}$ have been introduced at the AlGaAs/AlAs interfaces, i.e. the nodes of the optical mode. Figure 1 (a) shows a sketch and colored scanning electron microscopy image of the layer structure and doping concentration. Micropillars with a diameter of $20 \mu \mathrm{m}\left(r_{A}\right)$ were processed into the planar microcavity by a combination of optical lithography and reactive ion etching (see Fig. 1 (b)). Next, the sample was planarized with a polymer (benzocyclobutene) and a backside $\mathrm{n}$-contact was fabricated via a consecutive evaporation of a layer structure of AuGe-Ni-Au. The topside p-contacts consist of AuGe-Ni-Au and form a ring contact (see Fig. 1 (c)), which leaves an aperture of $10 \mu \mathrm{m}\left(r_{l}\right)$ for light collection. To improve homogeneity of current injection, a $10 \mathrm{~nm}$ layer of $\mathrm{Ti}-\mathrm{Au}$ has been deposited on the whole surface. Finally the gold contacts were manually contacted via a thermosonic ball-wedge- 


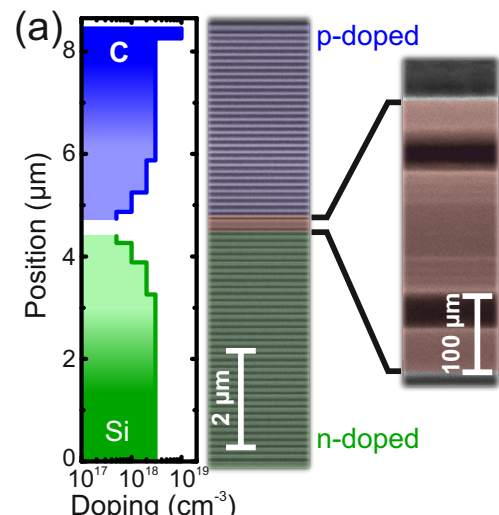

(d)
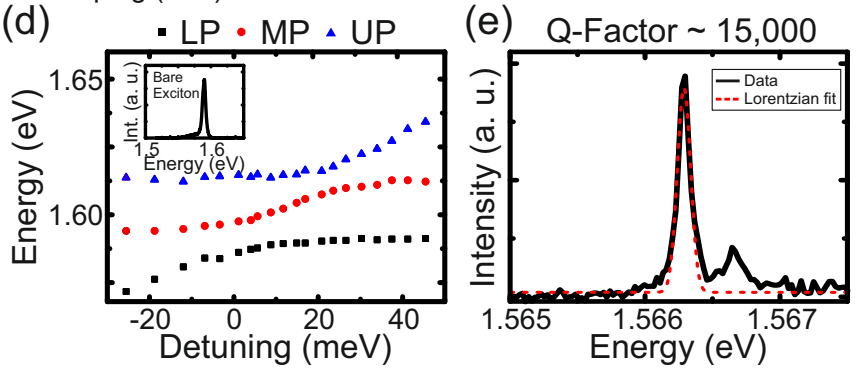

FIG. 1. (a) Sketch of the microcavity layer structure with the doping concentrations along the growth direction. (b) Scanning electron microscope image of an etched micropillar with a diameter of $20 \mu \mathrm{m}$. (c) Top-view white light image of the contacted micropillar device, the $10 \mu \mathrm{m}$ diameter aperture allows collection of the emitted light. (d) Strong coupling is evidenced by the characteristic anticrossing of the polaritonic resonances (heavy and light hole), mapped out via white light reflection. (e) Low power PL measurement of the quality factor of the device: A Lorentz fit of the ground state emission results in a $\mathrm{Q}$ factor exceeding 15,000.

\section{bonding technique.}

The optical characterization of the described structure was carried out by micro-photoluminescence at low pump powers at $5 \mathrm{~K}$ as well as white light reflection. The inset in Fig. 1 (d) shows the bare exciton emission of the QWstacks at $(1.5896 \pm 0.0005) \mathrm{eV}$ with a linewidth of $(4.4$ $\pm 0.5) \mathrm{meV}$ excited with a $532 \mathrm{~nm} \mathrm{cw}$ laser. A Lorentz fit of the ground state photoluminescence excited with a $745 \mathrm{~nm}$ pulsed-laser results in a Q-factor of the etched micropillars exceeding 15,000 (see Fig. 1 (e)), corresponding to a photon lifetime of approximately $6.3 \mathrm{ps}$. The differential resistance of the structure above threshold voltage was determined to be $12.0 \mathrm{k} \Omega$. By utilizing the naturally occuring wedge of the microcavity spacer thickness, we can map out a lower, middle and upper polariton branch. The normal mode splitting corresponding to the heavy-hole exciton yields $(11.0 \pm 0.5) \mathrm{meV}$ via spatially resolved white light reflection measurements (see Fig. $1(d)$ ). These parameters substantially exceed those of prior structures designed for electrical injection into polaritonic resonances ${ }^{15,16}$.

The following characterization of the electroluminescence, which is emitted from the microcavity, has been carried out via angle-resolved spectroscopy in the back Fourier plane imaging configuration at $15 \mathrm{~K}$. The slightly elevated temperatures above the liquid helium temperature of $4.2 \mathrm{~K}$ help to reduce the series resistance of our device, due to carrier activation. The current has been extracted via a voltage measurement at a serial resistor to the device and converted into the effective current density per micropillar. We use a combination of direct current injection and electrical pulses to increase polariton density inside the structure and minimize ohmic heating effects of the mirrors. To this end we employ a direct current below the threshold current of the diode at $1 \mathrm{~A} / \mathrm{cm}^{2}$. The angle resolved emission spectrum is displayed in Fig. 2 (a). The emission exhibits two clear branches, which we identify as the lower- and upper polariton branch. The lower branch further exhibits discretized energy levels due to the photonic confinement of the micropillar. We fit these branches with a coupled oscillator approach ${ }^{30}$, which yields a characterisitic Rabi-splitting of $7.5 \mathrm{meV}$ and a negative detuning of $-6.75 \mathrm{meV}$. The reduction of the Rabi-splitting, as compared to the white light reflection measurements is the result of the internal electrical field in the diode, which quenches the oscillator strength of the QW excitons.

To increase the carrier density, we apply rectangular pulses with a width of $10 \mathrm{~ns}$ and a repetition rate of $0.5 \mathrm{MHz}$, which corresponds to a $2 \mu$ s downtime of the structure to prevent charge buildup and heating. Figure 2 (b) to (d) present the emission properties with increased excitation current density. Each figure directly compares two current densities to highlight the continuous blueshift with increasing pump power. (b) depicts below threshold (c) at and above a first threshold and (d) at and above a second threshold. In (c) a prominent mode emerges in the discretized mode structure which we identify as the polariton condensate. The still strong background originates in the direct current emission. To quantify the emission properties, we have analysed the modes via a Lorentz fit of the ground state at $k=0 \mu \mathrm{m}^{-1}$. In Fig. 2 (e), we plot the input-output characteristics of our diode, revealing the occurrence of two thresholds which are marked by grey areas in the plot. This behaviour has been assigned in the literature to the polariton laser transition, driven by bosonic stimulation, as well as a laser transition above the Mott-density (conventional lasing, or BCS-type of lasing) $)^{28,29}$.

After the first threshold the intensity increases nonlinearly and we also observe a persisting blueshift, which aquires a logarithmic form. Further, the linewidth sharply drops by a factor of five at the first threshold, which is a clear sign of temporal coherence ${ }^{32}$. After the second threshold, the blueshift has saturated at a position slightly red shifted from the empty cavity mode, which was extracted from the the coupled oscillator fit. This incomplete blueshift can be attributed to carrier related changes of the refractive index. At the second threshold, the slope of the emitted intensity vs. excitation current increases again. We further observe an 
increase in linewidth, which is in qualitative agreement with previous experiments on optically injected polariton lasers $^{28}$.

Finally, we investigate the coherence properties of the device in more detail. We note, that while spatiotemporal coherence of optically injected polariton lasers and condensates has been widely investigated and discussed, direct measurements on electrically injected polariton lasers are still elusive. We have utilized a Michelson interferometer with a variable path length in the mirror-retroreflector configuration to study the coherence properties of our polariton laser. In the interferometer, the real-space image of the luminescence from the device is brought to overlap with its mirror-image on a beam splitter, and the interference picture is monitored on a CCD camera.

Figure 3 (a) and (c) shows an interferogram above $\left(1.15 \mathrm{P}_{\text {th }}\right)$ and below threshold $\left(0.50 \mathrm{P}_{\text {th }}\right)$ at a delay time of $\Delta t=0 \mathrm{ps}$ (path-length difference), where we can solely capture the autocorrelation signal for both excitations powers. For extended delay times of $\Delta t=5 \mathrm{ps}$ a pronounced interference pattern only develops for a power above the condensation threshold (Fig. 3 (b)), whereas no fringes can be observed below the threshold (Fig. $3(\mathrm{~d})$ ). The interference across the whole device shown in Fig. 3 (b) further implies a buildup of spatial coherence. Therefore the evaluation of the contrast $\left(\left(I_{\max }-I_{\min }\right) /\left(I_{\max }+I_{\min }\right)\right)$ as a function of the time delay between the two interferometer arms for a power of $1.15 \mathrm{P}_{\text {th1 } 1}$ is plotted in Fig. 3 (e). Above threshold, the interference contrast develops a characteristic damped, oscillatory pattern. The oscillation suggests, that at least two optical modes with a energetic separation of $790 \mu \mathrm{eV}$ contribute to the spectrum. This is in quantitative agreement with the observed multi-mode polaritonic lasing shown in Fig. 2 (c). From the damping of the oscillation, we can deduce a characteristic coherence time of $13.2 \mathrm{ps}$ for the ground-state of our condensate, which reflects a spectral linewidth of $\sim 0.1 \mathrm{meV}$. The fast, initial decay in the coherence measurement with a characteristic time constant of $\sim 7 \mathrm{ps}$ is a consequence of the excited state condensate, which is subject to a more pronounced dephasing. While strongly extended coherence times in optically driven polariton condensates, exceeding tens of picoseconds in e.g. Ref. 33 are reported in the literature, we believe that our result reflects a first important step towards the implementation of coherent electrically pumped polariton lasers.

We have implemented a vertically emitting GaAsbased electrically injected exciton-polariton laser based on a high quality factor GaAs microcavity without applying external magnetic fields or intra-cavity contacting in the lateral geometry. This device can be operated in close analogy to established optically pumped polariton lasers, featuring a clear nonlinearity in its input-output characteristics below the Mott transition, the persistent blueshift above threshold and a well-pronounced drop in the emission linewidth. Spatio-temporal coherence in our (a)

(b)
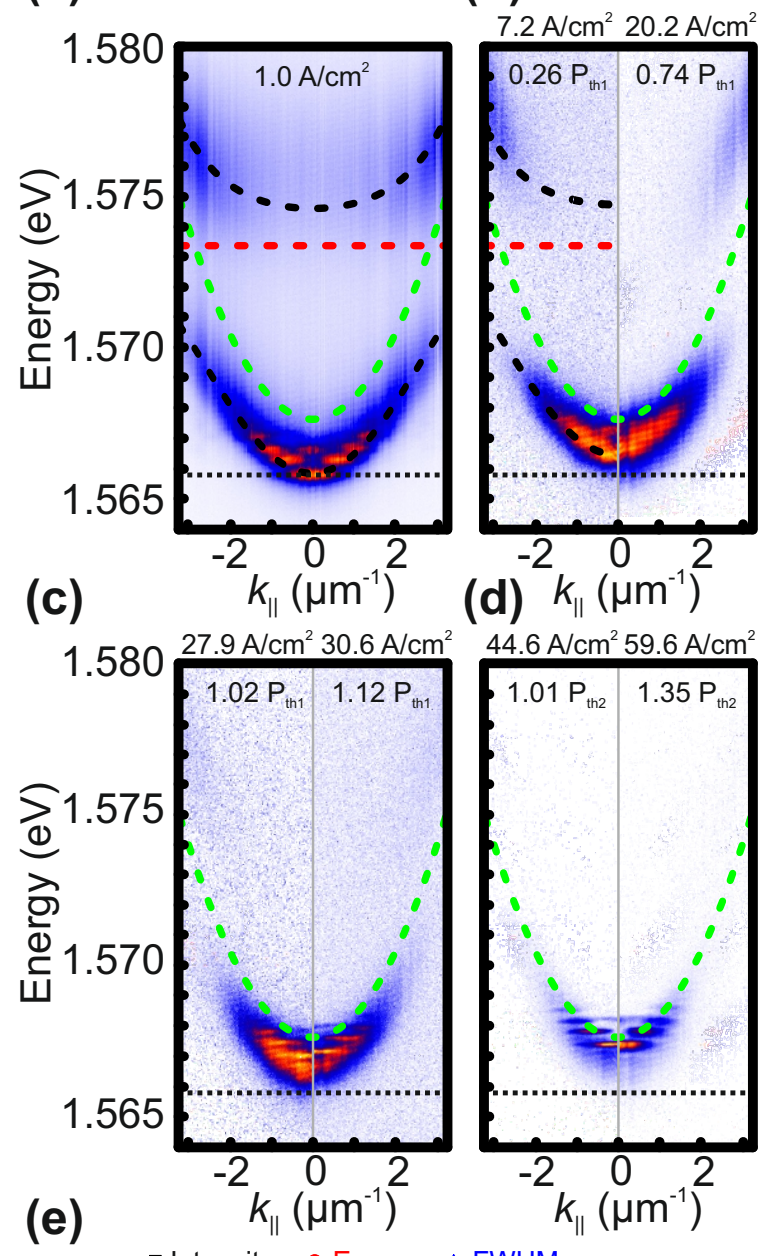

$44.6 \mathrm{~A} / \mathrm{cm}^{2} 59.6 \mathrm{~A} / \mathrm{cm}^{2}$
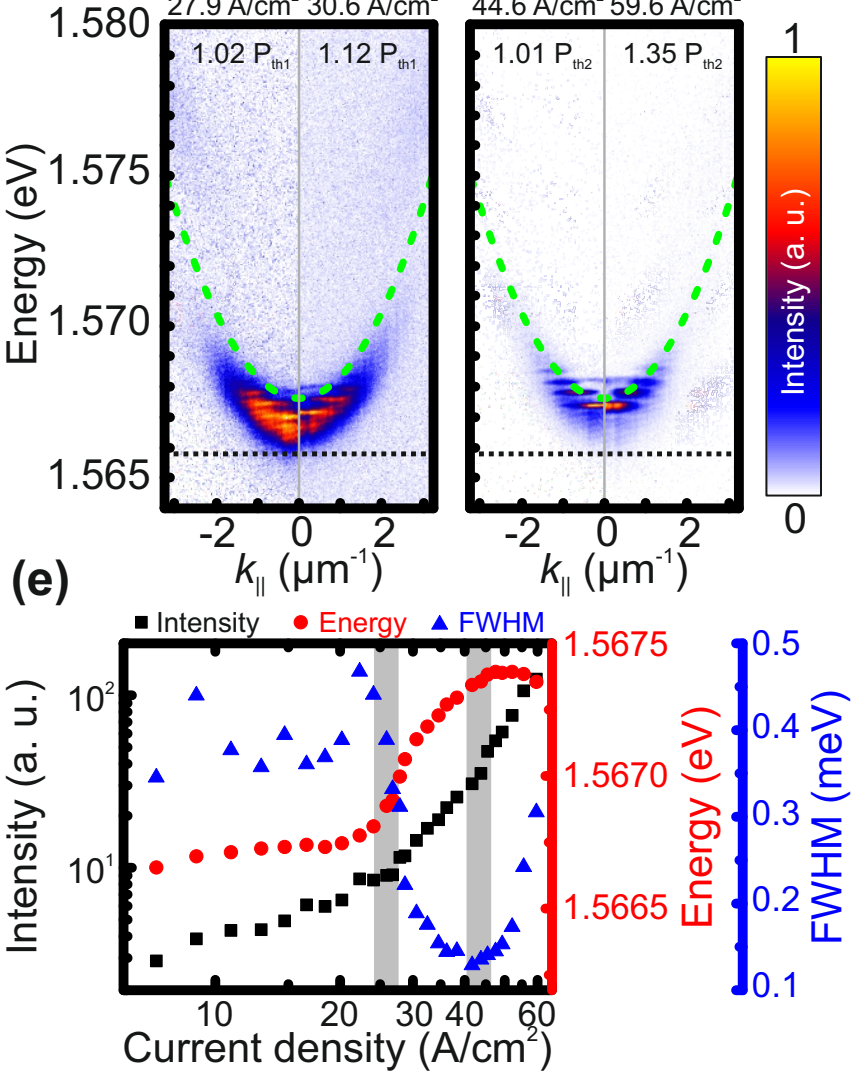

FIG. 2. (a) Momentum-resolved electroluminescence spectrum under direct current injection. Dashed lines correspond to a coupled oscillator model; black lower/upper polariton, green photon mode and red exciton. (b)-(d) Angleresolved electroluminescence series with increased pulsed current across the condensation threshold. We directly compare the positive/negative angle of different excitations to visualize changes in the dispersion relation. Note that (c) and (d) relate to the first and second threshold respectively. (e) Input-output analysis of the ground-state of the device with increased current, extracted from a Lorentz fit. We observe a blueshift of above $0.7 \mathrm{meV}$ after the first threshold, an energy pinning after the second and a linewidth drop at the first threshold. 
(a)

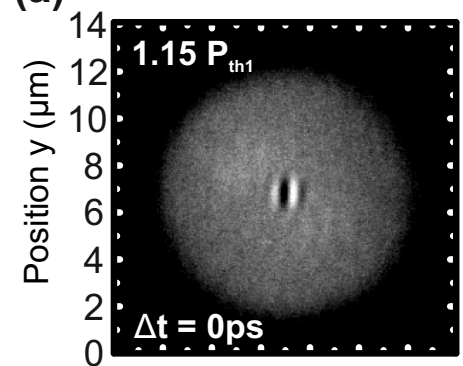

(c)

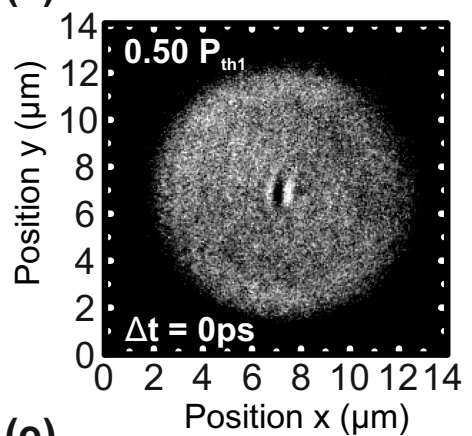

(e)

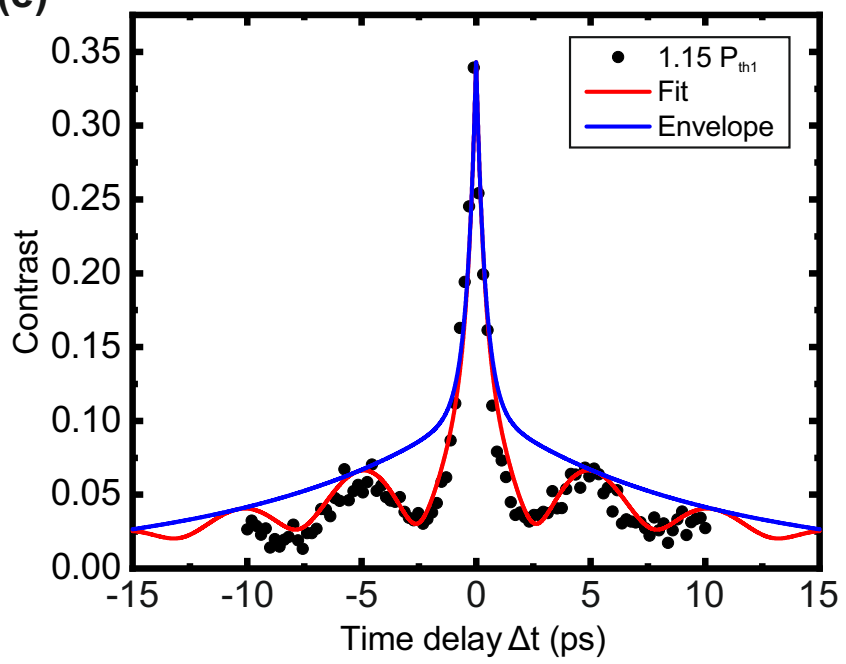

FIG. 3. Michelson interferometer (retroreflector configuration) of the electroluminescence from the aperture of the contacted micropillar at an injection current of $1.15 \mathrm{P}_{\text {th1 }}$ for (a) as well as (b) and $0.50 \mathrm{P}_{\mathrm{th} 1}$ for (c) as well as (d) at different delay times $\Delta t$ (path-length difference). Only at a power above the threshold a clear fringe pattern is observed for extended delay times, indicating spatial coherence of the emission. (e) Measurement of the temporal coherence for a power of 1.15 $\mathrm{P}_{\text {th1 }}$ showing the central fringe contrast vs. mirror delay. The oscillatory behaviour is a consequence of two beating modes, with a coherence of $13.2 \mathrm{ps}$ for the polaritonic ground state.

device directly evidenced via interference spectroscopy. While electrically injected polariton lasers are of interest towards the implementation of ultra-low power consuming coherent light-sources, recent advances in the implementation of electrical injection in polaritonic lattices ${ }^{34}$ hold great promise towards the implementation of elec- trically injected topological lasers and simulators based on bosonic stimulation ${ }^{20}$.

We gratefully acknowledge the financial support by the state of Bavaria, the DFG within the projects Schn13763.1 as well as KL3124/2-1 and the Würzburg-Dresden Cluster of Excellence on Complexity and Topology in Quantum Matter - ct.qmat. S.H. is grateful for funding received within the EPSRC Hybrid Polaritonics programme grant (EP/M025330/1).

Data available on request from the authors.

\section{REFERENCES}

${ }^{1}$ C. Weisbuch, M. Nishioka, A. Ishikawa, and Y. Arakawa, Phys. Rev. Lett. 69, 3314 (1992).

${ }^{2}$ J. Kasprzak, M. Richard, S. Kundermann, A. Baas, P. Jeambrun, J. M. J. Keeling, F. M. Marchetti, M. H. Szymanska, R. Andre, J. L. Staehli, V. Savona, P. B. Littlewood, B. Deveaud, and L. S. Dang, Nature 443, 409 (2006).

${ }^{3}$ H. Deng, G. Weihs, D. Snoke, J. Bloch, and Y. Yamamoto, PNAS 100, 15318 (2002).

${ }^{4}$ P. Tsotsis, P. S. Eldridge, T. Gao, S. I. Tsintzos, Z. Hatzopoulos, and P G Savvidis, New J. Phys. 14, 023060 (2012).

${ }^{5}$ A. Dreismann, H. Ohadi, Y. Redondo, R. Balili, Y. Rubo, S. Tsintzos, G. Deligeorgis, Z. Hatzopoulos, P. G. Savvidis, and J. J. Baumberg, Nat. Mater. 15, 1074 (2016).

${ }^{6}$ D. Sanvitto, and S. Kena-Cohen, Nat. Mat. 15, 1061 (2016).

${ }^{7}$ M. D. Fraser, Semicond. Sci. Technol. 32, 093003 (2017).

${ }^{8}$ S. Christopoulos, G. Baldassarri Höger von Högersthal, A. J. D. Grundy, P. G. Lagoudakis, A. V. Kavokin, J. J. Baumberg, G. Christmann, R. Butte, E. Feltin, J. Carlin, and N. Grandjean, Phys. Rev. Lett. 98, 126405 (2007).

${ }^{9}$ J. D. Plumhof, T. Stoferle, L. Mai, U. Scherf, and R. F. Mahrt, Nat. Mat. 13, 247 (2014).

${ }^{10}$ K. S. Daskalakis, S. A. Maier, R. Murray, and S. Kena-Cohen, Nat. Mater. 13, 271 (2014).

${ }^{11}$ N. Lundt, S. Klembt, E. Cherotchenko, S. Betzold, O. Iff, A. V. Nalitov, M. Klaas, C. P. Dietrich, A. V. Kavokin, S. Höfling, and C. Schneider, Nat. Commun. 7, 13328 (2016).

${ }^{12}$ C. P. Dietrich, A. Steude, L. Tropf, M. Schubert, N. M. Kronenberg, K. Ostermann, S. Höfling, and M. C. Gather, Sci. Adv. 2, 1600666 (2016)

${ }^{13}$ G. G. Paschos, N. Somaschi, S. I. Tsintzos, D. Coles, J. L. Bricks, Z. Hatzopoulos, D. G. Lidzey, P. G. Lagoudakis and P. G. Savvidis, Sci. Rep. 7, 11377 (2017).

${ }^{14}$ S. I. Tsintzos, N. T. Pelekanos, G. Konstantinidis, Z. Hatzopoulos, and P. G. Savvidis, Nature 453, 372 (2008).

${ }^{15}$ C. Schneider, A. Rahimi-Iman, N. Y. Kim, J. Fischer, I.G. Savenko, M. Amthor, M. Lermer, A. Wolf, L. Worschech, V. D. Kulakovskii, I. A. Shelykh, M. Kamp, S. Reitzenstein, A. Forchel, Y. Yamamoto, and S. Höfling, Nature 497, 348 (2013).

${ }^{16}$ P. Bhattacharya, B. Xiao, A. Das, S. Bhowmick, and J. Heo, Phys. Rev. Lett. 110, 206403 (2013).

${ }^{17}$ M. Baten, P. Bhattacharya, T. Frost, S. Deshpande, A. Das, D. Lubyshev, J. Fastenau, and A. W. K. Liu, Appl. Phys. Lett. 104, 231119 (2014).

${ }^{18}$ P. Bhattacharya, T. Frost, S. Deshpande, M. Baten, A. Hazari, and A. Das, Phys. Rev. Lett. 112, 236802 (2014).

${ }^{19}$ C. Schneider, K. Winkler, M. D. Fraser, M Kamp, Y. Yamamoto, E. A. Ostrovskaya and S. Höfling, Rep. Prog. Phys. 80, 1 (2016).

${ }^{20}$ P. St-Jean, V. Goblot, E. Galopin, A. Lemaitre, T. Ozawa, L. Le Gratiet, I. Sagnes, J. Bloch, and A. Amo, Nat. Photon. 11, 651 (2017).

${ }^{21}$ S. Klembt, T.H. Harder, O.A. Egorov, K. Winkler, R. Ge, M.A. Bandres, M. Emmerling, L. Worschech, T.C.H. Liew, M. Segev, C. Schneider, S. Höfling, Nature (2018).

${ }^{22}$ A. Verger, C. Ciuti, and I. Carusotto, Phys. Rev. B 73, 193306 (2006). 
${ }^{23}$ T. C. H. Liew and V. Savona, Phys. Rev. Lett. 104, 183601 (2010).

${ }^{24}$ M. Bamba, A. Imamoglu, I. Carusotto, and C. Ciuti, Phys. Rev. A 83, 021802 (2011).

${ }^{25}$ H. Flayac and V. Savona, Phys. Rev. A 96, 053810 (2017).

${ }^{26}$ G. Munoz-Matutano, A. Wood, M. Johnsson, X. Vidal, B. Q. Baragiola, A. Reinhard, A. Lemaitre, J. Bloch, A. Amo, G. Nogues, B. Besga, M. Richard, and Thomas Volz, Nat. Mater. 18, 213 (2019).

${ }^{27}$ A. Delteil, T. Fink, A. Schade, S. Hö̈ling, C. Schneider, A. Imamoglu, Nat. Mater. 18, 219 (2019).

${ }^{28}$ D. Bajoni, P. Senellart, E. Wertz, I. Sagnes, A. Miard, A. Lemaître, and J. Bloch, Phys. Rev. Lett. 100, 047401 (2008).

${ }^{29}$ J. Hu, Z. Wang, S. Kim, H. Deng, S. Brodbeck, C. Schneider, S. Höfling, N. H. Kwong, R. Binder, arXiv:1902.00142 (2019).
${ }^{30}$ A. Kavokin, J. J. Baumberg, G. Malpuech, and F. P. Laussy, Microcavities (Oxford University Press, Oxford, 2011).

${ }^{31}$ b C. Ciuti, V. Savona, C. Piermarocchi, A. Quattropani, and P. Schwendimann, Phys. Rev. B 58, 7926 (1998).

${ }^{32}$ N. Wiener, Time Series. M.I.T. Press, Cambridge, Massachusetts. p. 42 (1964).

${ }^{33}$ A. P. D. Love, D. N. Krizhanovskii, D. M. Whittaker, R. Bouchekioua, D. Sanvitto, S. Al Rizeiqi, R. Bradley, M. S. Skolnick, P. R. Eastham, R. André, and Le Si Dang, Phys. Rev. Lett. 101, 067404 (2008).

${ }^{34}$ H. Suchomel, S. Klembt, T. H. Harder, M. Klaas, O. A. Egorov, K. Winkler, M. Emmerling, R. Thomale, S. Höfling, and C. Schneider, Phys. Rev. Lett. 121, 257402 (2018). 